\title{
In-Flight Acoustic Testing Techniques Using the Y0-3A Acoustic Research Aircraft
}

\section{J.L. Cross and M.E. Watts}

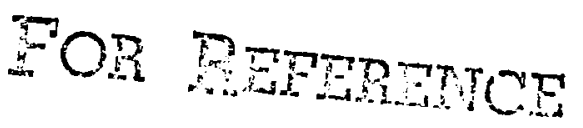

February 1984

HOT TO BE TAKEI FROM TIIS MOO:

\section{LBRARV BgPY}

VUARG 1984

TANGLEY RESEARCH CENTER

LIERARY, NASA

HAMBPTOIN YIRGINIA' 


\section{In-Flight Acoustic Testing Techniques Using the Y0-3A Acoustic Research Aircraft}

J. L. Cross

M. E. Watts, Ames Research Center, Moffett Field, California

\section{N/SA}

National Aeronautics and

Space Administration 
IN-FLIGHT ACOUSTIC TESTING TECHNIQUES USING THE YO-3A ACOUSTIC RESEARCH AIRCRAFT

\author{
Jeffrey L. Cross ${ }^{*}$ and Michae1 E. Watts ${ }^{*}$ \\ Ames Research Center, NASA, \\ Moffett Field, California
}

\section{Abstract}

This report discusses the flight testing techniques and equipment employed during air-toair acoustic testing of helicopters at Ames Research Center. The in-flight measurement technique used enables acoustic data to be obtained without the limitations of anechoic chambers or the multitude of variables encountered in ground based flyover testing. The air-to-air testing is made possible by the NASA YO-3A Acoustic Research Aircraft. This "Quiet Aircraft" is an acoustical1y Instrumented version of a quiet observation aircraft manufactured for the military. To date, tests with the following aircraft have been conducted: Y0-3A background noise; Hughes 500D; Hughes AH-64; Bell AH-1S; Bell AH-1G. Several system upgrades are being designed and implemented to improve the quality of data. This report will discuss not only the equipment involved and aircraft tested, but also the techniques used in these tests. In particular, formation flying, position locations, and the test matrices will be discussed. Examples of data taken will also be presented.

\section{Nomenclature}

$\begin{array}{ll}\mathbf{C}_{t} & \text { thrust coefficient } \\ \mathbf{g} & \text { gravitational constant } \\ \mathrm{L} & \text { rotor 1ift } \\ \mathrm{M}_{\mathrm{t}} & \text { advancing tip Mach number } \\ \mathbf{r} & \text { rotor radius } \\ \mathrm{R} & \text { gas constant } \\ \mathrm{T} & \text { outside air temperature } \\ \mathrm{V} & \text { aircraft velocity } \\ \gamma & \text { specific heat ratio } \\ \mu & \text { advance ratio } \\ \rho & \text { density } \\ \sigma & \text { rotor solidity } \\ \Omega & \text { rotational velocity }\end{array}$

\section{Introduction}

Flight testing is an inherent part of aerospace engineering. Every facet of aircraft design is at one time or another flight tested to determine its true "real world" characteristics. Many of these facets are relatively simple to measure In a flight environment while others provide obstacles which are on $1 y$ recently being overcome. One such area is acoustics of helicopters. While it is true that air-to-ground and internal noise analysis have been performed for many years, it is only recently that air-to-air acoustics tests have been attempted. These have been in response to the increasing concern over helicopter noise levels. In response to these concerns, NASA and the Army have had an ongoing acoustic research program in the area of helicopter noise for many years. As research progressed, it became evident that to fully understand the various noise genera-

\footnotetext{
* Aerospace Engineer, Member AIAA.
}

tion processes involved, the methods used in obtaining the noise data were inadequate and a different method of testing would be necessary. This realization was brought about because the two main sources of impulsive noise in helfcopters are blade vortex interaction and shock effects. As both of these occur almost entirely in forward flight, a means of accurately measuring these generation processes was needed.

Methods used to measure helicopter noise included: ground based microphone arrays; externally and internally mounted microphones; and wind tunnel tests. Each of these methods provided good data in certain areas, but they all have drawbacks which point to the necessity of measuring noise in an airborne fixed relative reference frame. Drawbacks for the other methods include:

1. reflected noise interference from the ground or other surfaces

2. doppler effects caused by relative speeds between source and microphone

3. airframe attenuation for internally mounted microphones

4. near field effects interfering with far field measurements

5. atmospheric effects caused by altitude differences in flyover testing.

To eliminate the other effects, a method of measurement was needed which caused the microphones to be traveling in the far field with the same velocity as the aircraft being measured, and away from any reflective surfaces. The answer was to mount a microphone on an aircraft which would fly at the same speed as the helicopter. This was done on the OV-1 Mohawk aircraft in 1975 by the Army (references 1 and 2). While this eliminated the problems listed above, it added the problem of background noise overwhelming the noise being measured.

In general, the background notse of the Mohawk would mask the helicopter noise. However, if the specific frequencies being investigated were known, the engines could be tuned to seperate the Mohawk blade passage and engine noise for those frequencies. This worked satisfactoraly if specific noise data were being sought, but was cumbersome if general notse data was the objective. Also, with a stall speed of 80 knots, the Mohawk was not well suited for measuring blade vortex interaction effects which are most prominent in slower flight. This brought about the instrumentation and use of a YO-3A "Quiet" aircraft belonging to the Federal Bureau of Investigation in 1976 for air-to-air acoustic testing. The success of this testing led to NASA's acquisition of a Yo-3A dedicated to in-flight acoustic research. This report deals with the present and 
future role of this aircraft in the ongoing effort by NASA to understand the noise generation processes involved in helicopter flight. It will also describe the aircraft, instrumentation involved, and several tests which have already been conducted. Sample data will be presented from each of the other tests where available.

\section{Equipment}

The YO-3A Acoustic Research aircraft, shown in figure 1, is used by NASA Ames for in-flight measurement of aircraft noise, particularly helicopter noise.

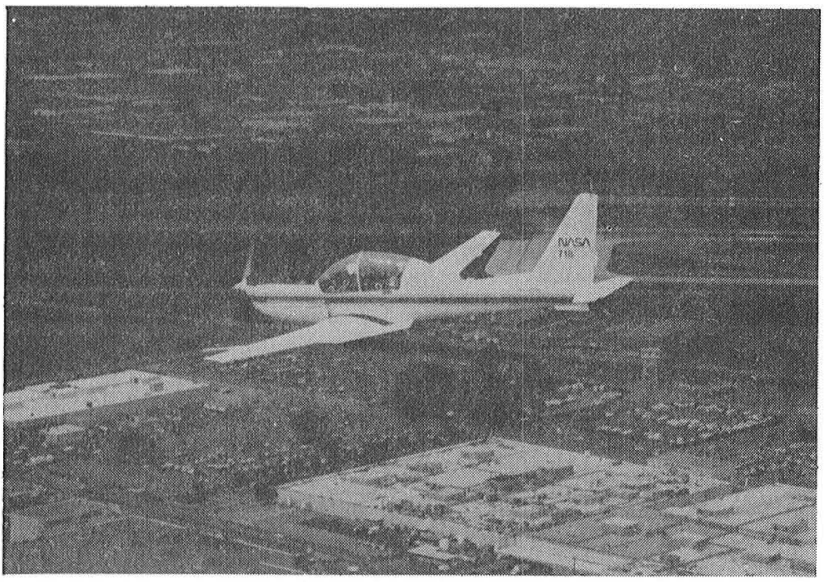

Fig. 1 Yo-3A Acoustic research aircraft.

Lockheed Missles and Space Company of Sunnyvale, California designed and built the Yo-3A for the United States Army in 1969. Origina11y, the aircraft's mission was low speed, low altitude observation of enemy troop and supply movements during the Vietnam War. The YO-3A, and its predecessors the QT-2 and Q-Star are based on the Schweizer SGS 2-32 sail plane, figures 2 and 3 respectively.

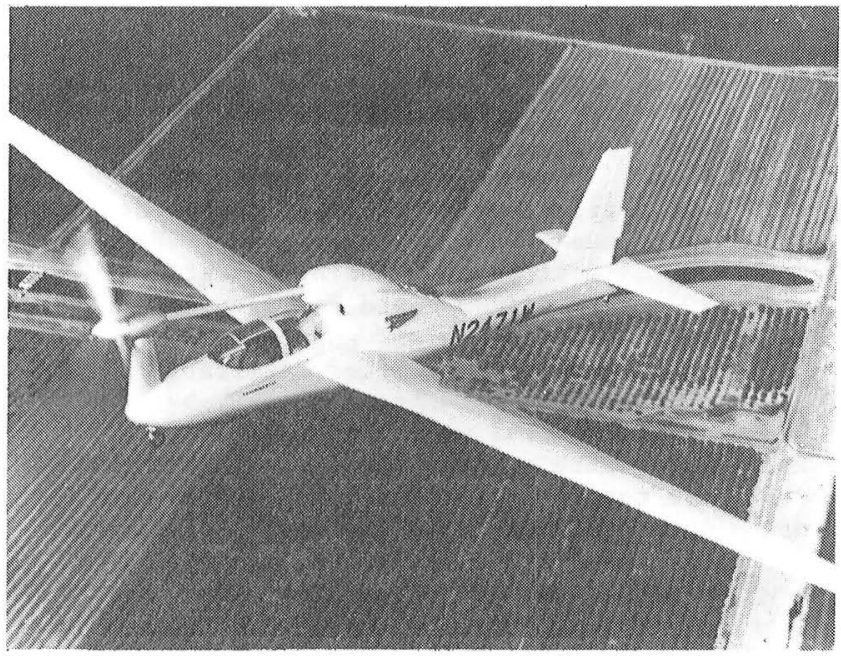

Fig. 2 QT-2 aircraft.

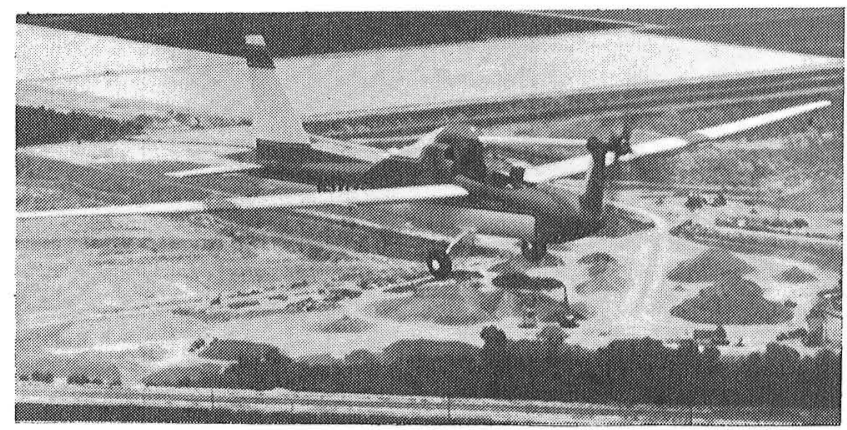

Fig. 3 Q-Star aircraft.

The physical characteristics of the YO-3A are given in table 1.

Table 1 Y0-3A Physica1 Characteristics

$\begin{array}{lr}\text { Wing Span } & 57 \mathrm{ft} \\ \text { Length } & 29.3 \mathrm{ft} \\ \text { Height } & 9.1 \mathrm{ft} \\ \begin{array}{l}\text { Maximum Gross } \\ \text { Takeoff Weight }\end{array} & 3800 \mathrm{lbs} \\ \begin{array}{l}\text { Power P1ant, } \\ \quad \text { Continental }\end{array} & 210 \mathrm{hp} \\ \begin{array}{l}\text { Propeller Diameter } \\ \text { Stal1 Speed, }\end{array} & 100 \mathrm{in} \\ \quad \text { with Flaps } & 60 \text { knots } \\ \text { Maximum Speed } & 110 \text { knots } \\ \text { Crew } & 2\end{array}$

A Continental reciprocating engine drives a three bladed variable pitch propeller. The blades, wood laminates hand crafted by ole Fahlen, have an engine to propeller speed reduction of 3 to 1. The low propeller tip speed, about $360 \mathrm{fps}$, is one of the two key design features leading to the extraodinary quietness of. the YO-3A. The other quieting feature is a muffler specifically tailored to the engine and is mounted under a prominent starboard cowling running the length of the fuselage. The aircraft, while extremely quiet, as would be expected has limited performance. Its maximum level flight airspeed is one hundred and ten knots while its stall speed is sixty knots. The maximum rate of climb is approximately five hundred feet per minute. The Y0-3A has a crew of two, seated in tandem. The pilot is seated in the rear seat with the test engineer in front with its expansive view and specially laid out instrument panel.

The aircraft sensors specially installed for flight testing consist of an instrument boom and radio links. The instrument boom, mounted off the port wing, contains sensors that measure static and dynamic pressure, angle of attack, side slip, and outside aix temperature. The pilot instruments for airspeed, altitude and rate of climb are obtained off a seperate pitot-static tube, mounted on the starboard wing. The radio links are an IRIG-B time code reciever used for data time correlations and a one per revolution signal broadcast from the test helicopter. This one per rev signal is obtained from an optical sensor mounted in the fixed helicopter system. The sensor system uses a small piece of reflective tape, adhered to the rotating system onboard the helicopter, to sense each complete revolution of the main rotor 
shaft. The signal from the optical sensor is conditioned for improved signal to noise ratio during transmission to the YO-3A. The complete system is compact and easily installed on any he1icopter to be tested with the Y0-3A. Correlation of the acoustic data with the helicopter blade azimuth position is performed through the use of this one per rev signal.

Acoustic instrumentation on the YO- $3 \mathrm{~A}$ consists of two modified dual channel microphone power supplies, three microphones with preamp1ifiers, and an oscilloscope. The power supplies have been specially modified in three ways: to run off of the 28 volt DC aircraft power; a factory modification which allows a $-20,-10,0$, 10 , or 20 decibe1 (db) gain on each output signal; and (3) a potentiometer was added which allows minute gain adjustments during calibration. These drive the three one half-inch preamplifiers and their attached one half inch condenser microphones with one channel being left as a spare. A portable oscilloscope is used for the on line monitoring of in-flight data as it is recorded. The microphone signals and aircraft sensors mentioned above are recorded along with a voice track of the intercom and radio comments on a 14 track FM wide band airborne tape recorder.

The flight engineer's station is located in the front seat of the YO- $3 \mathrm{~A}$ and his instrumentation panel layout is shown in figure 4.

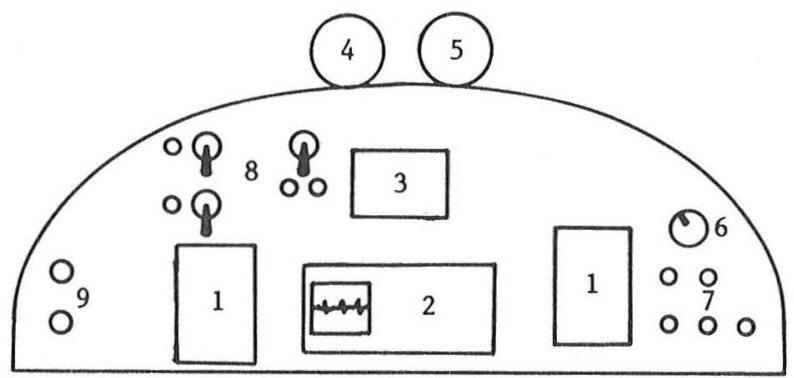

1. Microphone power supplies

2. Oscilloscope

3. Digita1 Temerature gage

4. Airspeed gage

5. Altimeter

6. Rotary switch controlling input to 2

7. Power and data on/off switches

8. Calibration switches

9. Leads to recorder

Fig. 4 Flight test engineers panel layout.

The flight engineer has the ability, through the rotary switch located to the upper right of the pane1, to moniter any of the three microphone signals, the time code, or the 1 per rev signals on the centrally mounted oscilloscope. The microphones and preamps are mounted on aerodynamic struts on each wingtip and the top of the vertical tail, as show in figure 5 .

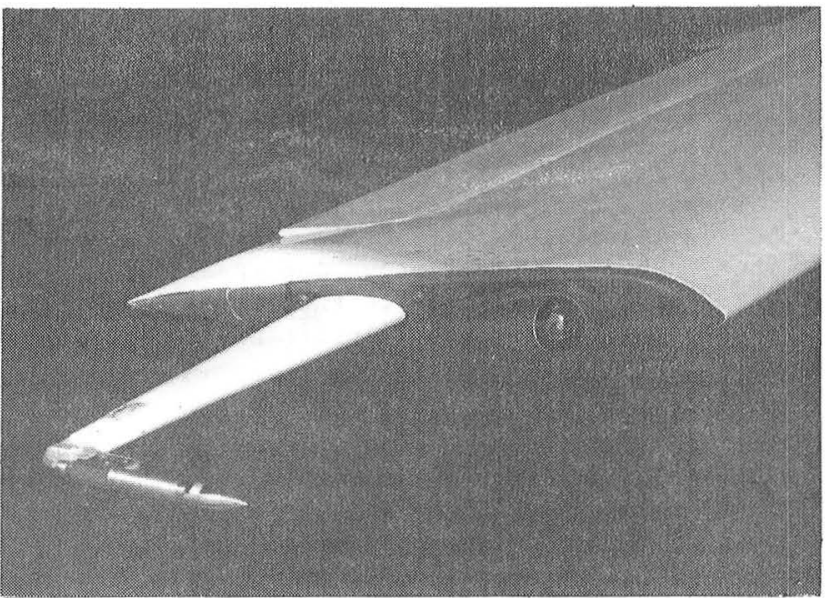

Fig. 5 Microphone mounting strut.

In an effort to reduce wind noise, the wires from the mierophones are kept out of the airstream by passing them through the body and wings. In addition, bullet nose covers are used on the microphones to reduce noise and protect the microphones. Calibration of the acoustic equipment is done on each test tape with a $124 \mathrm{db}, 250 \mathrm{~Hz}$ pistonphone. The calibration serves many purposes: adjustment of each microphone channel to the desired output setting with the potentiometer modification discussed above; obtaining reference values for conversion to engineering units; and a functional check of each microphone channel before and after each flight.

\section{Testing Techniques}

Acoustic flight testing of helicopters with the Y0-3A has led to the developement and/or utilization of many unique flight testing techniques These techniques include real time test point calculation, formation flying, and aircraft positioning techniques. Many different testing techniques have been investigated over the past years of in-flight testing.

The most offensive helicopter radiated noise propagates ahead of the aircraft since it is being generated on the advancing blade. To concentrate on this noise, the YO-3A flies as lead aircraft during in-flight testing. During the initial testing of the in-flight concept, done jointly by the U.S. Army Air Mobility R\&D Laboratory and the Army Engineering Flight Activity at Edwards Air Force Base, California, tests were done to locate the predominant directivity paths. These tests were accomplished by having the test helicopter fly an arc around the acoustic recording aircraft (initially an OV-1 Mohawk). A typical test point would be for the helicopter to begin a set distance behind the recording aircraft and fly to the side maintaining a constant radial distance. A motion picture camera onboard the test helicopter photographed the recording aircraft to aid in 
determining the relative aircraft positions at maximum sound intensity. A matrix of horizontal and vertical sweeps were carried out with a UH-1H Iroquols (Huey) as the test helicopter. The test results, when compared to theoretical predictions, showed two prime locations for capturing the most severe noise source. The first, measuring high Mach effects on the advancing blade, was for the rotor hub to be coplanar with the tail microphone on the recording aircraft. The second, measuring blade vortex interaction, was for the helicopter to be thirty degrees above the recording aircraft and forty five degrees to the aft of portside. These test points are illustrated in figure 6 .

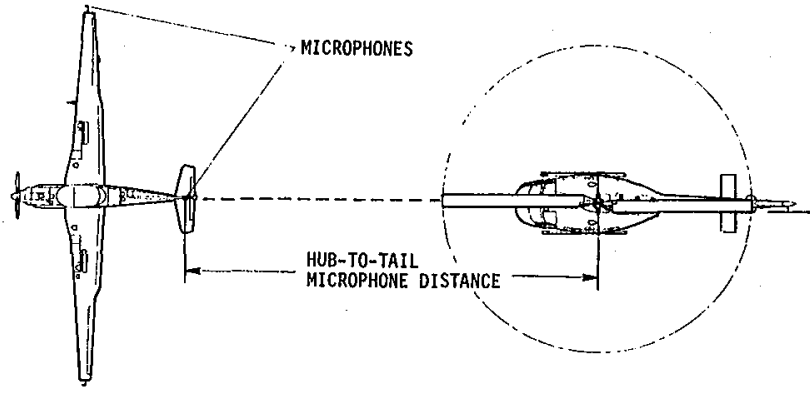

(a) Trail formation

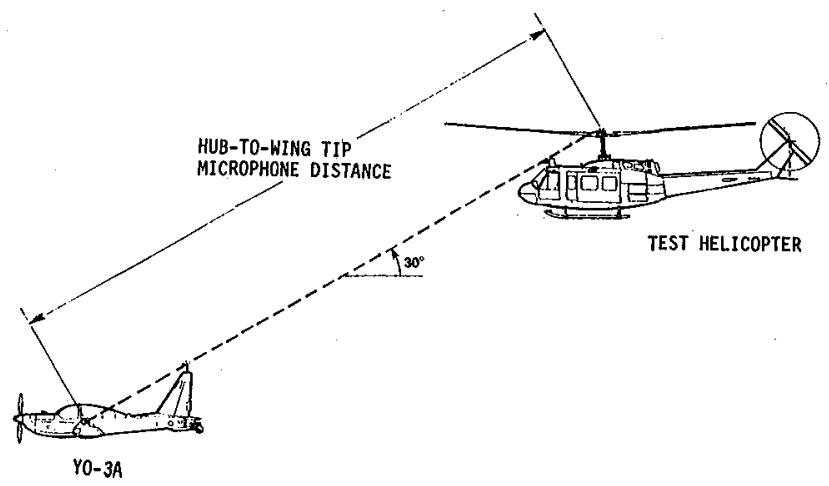

(b) Left formation

Fig. 6 Formation flight positions.

Since these initial tests, two more positions have been added to the desired test locations. Several wind tunnel tests (reference 3 ) have shown an acoustic peak when the test rotor is thirty degrees above the microphone plane and forty five degrees aft of starboard. The second location is actually a position sweep. This begins aft of the test aircraft, as in the trail position, and flies an arc to a point directly overhead of the YO-3A. This is done to identify any significant deviations from the blade vortex interaction characteristics of the two bladed UH- $1 \mathrm{H}$.

These test locations form one $1 \mathrm{eg}$ of the three-dimensional test matrices that are used during acoustic testing. The remaining two legs are rate of descent and airspeed. These, of course, depend on the YO-3A's flight envelope. As mentioned earlier, the top speed of the YO-3A in level flight is $110 \mathrm{knots}$, but it has a top speed of 130 knots in a dive. Table 2 shows a typica1 test matrix consisting of fifty six data points.
Table 2 Acoustic Test Matrix

\begin{tabular}{|c|c|c|c|c|c|c|c|}
\hline \multicolumn{8}{|c|}{ Alrspeed, knots } \\
\hline \multirow{2}{*}{$\begin{array}{c}\text { Rate } \\
\text { of Sink } \\
\text { fps }\end{array}$} & 60 & 80 & 90 & 100 & 110 & 120 & 130 \\
\hline & & & & & & & \\
\hline 0 & LTR & LTE & TR & LTE & $\mathbf{T}$ & - & - \\
\hline 200 & LT & LT & LT & LR & LT & - & - \\
\hline 400 & LR & LR & LTR & LTE & LR & TR & - \\
\hline 600 & L & LR & L & TR & LT & LT & - \\
\hline 800 & LR & LR & LR & L & LT & $\mathbf{T}$ & $\mathrm{T}$ \\
\hline 1000 & - & - & - & - & - & - & $\mathrm{T}$ \\
\hline & & & & $\begin{array}{l}L= \\
T= \\
R=\end{array}$ & $\begin{array}{l}\text { eft } \\
\text { rail } \\
\text { ight }\end{array}$ & $\begin{array}{l}\text { Posi } \\
\text { Posi }\end{array}$ & \\
\hline
\end{tabular}

The aircraft separation and positioning are maintained visually using a hand held rangefinder by the test helicopter co-pilot/test engineer to establish and maintain the proper distance. The proper left and right positioning behind the YO-3A is judged by the pilot, but during the practice runs to familiarize the test team with the "feel" of these positions, the proper relative vertical seperation is generally assisted by observation from the chase aircraft. The range finder is generally useful in keeping a constant distance thru forty degrees elevation, but then pilot proficiency is relied on to hold the radius for the remainder of the point. Again, comments from the chase aircraft during these practice runs, are invaluable. When proficiency is obtained in holding the formations, the chase aircraft moves to a distance where its noise will not contribute to the test data. These visual techniques of formation flying have been found to be accurate to within $+/-3$ feet of the desired distances.

Test procedures call for the Y0-3A pilot to set up on the specified airspeed and altitude of the test condition. When stable, the helicopter pilot maneuvers into place using the techniques discussed above. Once the proper formation is obtained in level flight, the copilot/test engineer notifies the test engineer in the $\mathrm{YO}-3 \mathrm{~A}$ by switching on the one per rev signal. While the test helicopter is setting up, the Yo-3A test engineer adjusts the $d b$ gain settings to maximize the microphone response, but still avoiding signal saturation. When the one per rev signal is recleved, the test engineer begins taking data, all the while monitoring the acoustic levels, one per rev, and time code on the oscilloscope. Each data record begins with comments denoting the test condition and run number, and ends with pilot comments. The Yo-3A pilot comments on the airspeed, altitude, and consistancy of the run, the helicopter pilot comments on the positioning of the aircraft and control inputs needed to maintain that position. These comments prove to be invaluable during data reduction in helping select the best section of data to use.

These procedures work well for the level flight test points. However during the higher rates of sink point, where timing is critical, refinements of this technique are required. For these points the YO-3A pilot sets up on the air- 
speed with the test aircraft in close formation but not trying to hold position. Then with a radio call, the Y0-3A pilot noses over to obtain and maintain the specified rate of $B$ ink while holding airspeed. The helicopter pilot simultaneously tries to get into proper position. As with level flight, the one per rev signal is used to indicate that the helicopter is in position. When the test engineer is recieving the one per rev and has been informed by his pilot that they are stable, the data record is begun. Since each test point lasts for a minimum of thirty seconds of data, significant altitude can be lost if the set up is not quickly accomplished.

The test matrix discussed above is flown in such a way as to maintain several nondimensional engineering parameters constant: coefficient of thrust; advancing tip Mach number; and advance ratio. By keeping these parameters constant, any acoustic differences between test points must be due to the remaining variables (e.g. rate of descent). Thrust coefficient, defined in equation 1 ,

$$
\mathrm{C}_{\mathrm{t}} / \sigma=\mathrm{L} /\left(\rho \pi \mathrm{r}^{4} \Omega^{2} \sigma\right)
$$

is kept constant by varying altitude, keeping track of fuel used and by assuming rotor thrust equals aircraft gross weight. At each data point, a test altitude must be calculated and held, but during a rate of sink test point the data is begun such that the desired altitude is approximately the mean of that run. The advancing tip Mach number and the advance ratio, equations 2 and 3

$$
\begin{aligned}
& \dot{M}_{t}=V / \sqrt{\gamma g R T} \\
& \mu=V / \Omega R
\end{aligned}
$$

respectively, are obtained by varying airspeed and main rotor rotational speed. These computations cannot be done a priori as neither the fuel use profile nor outside air temperture can be adequately forecast. A routine has been written and programmed into a portable programmable calculator which computes these flight parameters in real time by an observer on the chase aircraft. This minimizes the number of people involved in the test, does not restrict the test area, and maintains a safe number of eyes outside the cabins for air traffic identification and avoidance.

\section{Tests Conducted}

A total of six flight test programs have been conducted utilizing the Y0-3A aircraft. The helicopters tested by AVRADCOM, before NASA received a Yo-3A dedicated to acoustic testing, were the $\mathrm{UH}-1 \mathrm{H}, \mathrm{UH}-60, \mathrm{UH}-61, \mathrm{AH}-63$, and $\mathrm{AH}-64$. Tests performed by NASA, either in cooperation with or independent of AVRADCOM, will be discussed in this section.

Knowledge of the characteristics of an experimenters equipment is vital to the accurate interpretation of data obtained with that equipment. To that end, a background noise test was flown with the Yo-3A in 1982. Speed runs from 60 to 110 knots in level flight and descent rates of up to 1000 feet per minute at various airspeeds were flown. In addition, several runs were made while deploying the spoilers and with idle engine to try and determine how much of an effect these had on the background noise levels. Narrow band graphs of a high and low speed case are presented in figure 7.

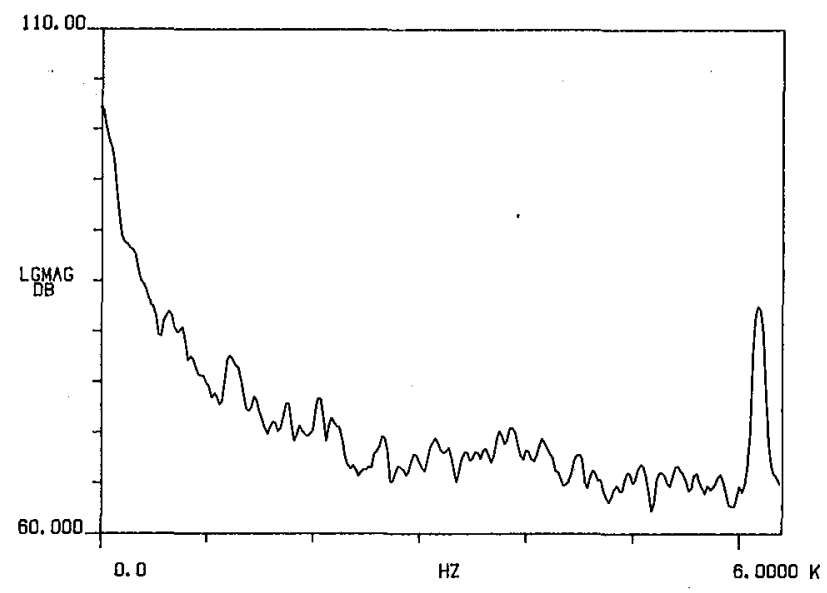

(a) 108 knots airspeed

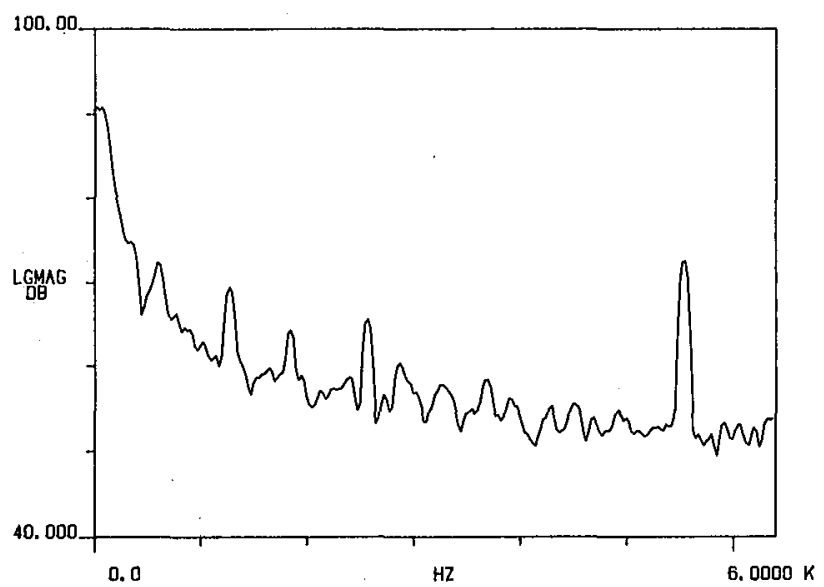

(b) 60 knots airspeed

Fig. 7 YO-3A background noise.

In 1980 , a test involving a Hughes $500 \mathrm{D}$ was flown at NASA Ames Research Center. The purpose of this test was to investigate, both by flyover and air-to-air testing, the benefits in noise reduction which a four bladed tail rotor produced over the standard two bladed configuration.

Analysis of the air-to-ground data, provided by Hughes helicopters, has shown an average of $3 \mathrm{db}$ reduction between the two and four bladed configurations over an airspeed range of 65 to 130 knots with the maximum reduction of $5.4 \mathrm{db}$ occurring at 80 knots. Figure 8 shows narrow band data for the air-to-air test at this maximum reduction speed of 80 knots. 


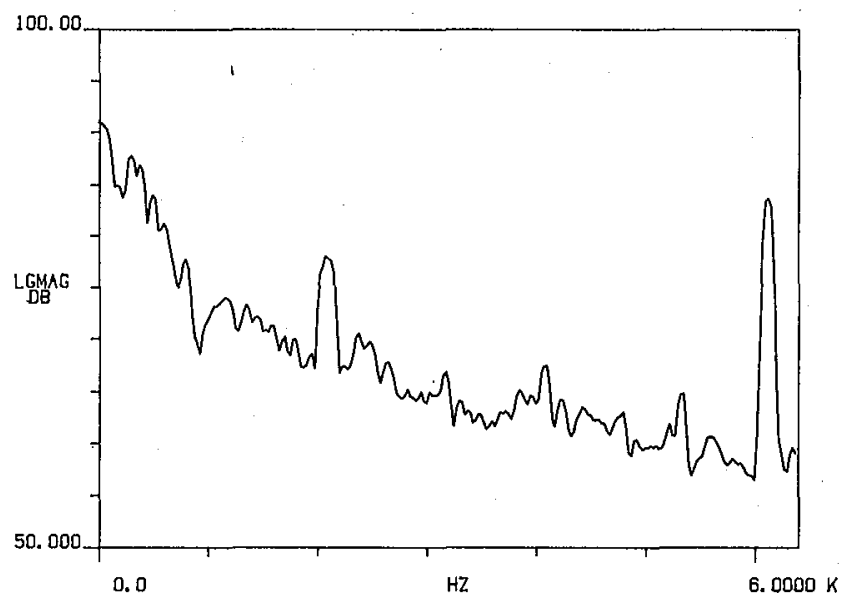

(a) Two bladed tail rotor

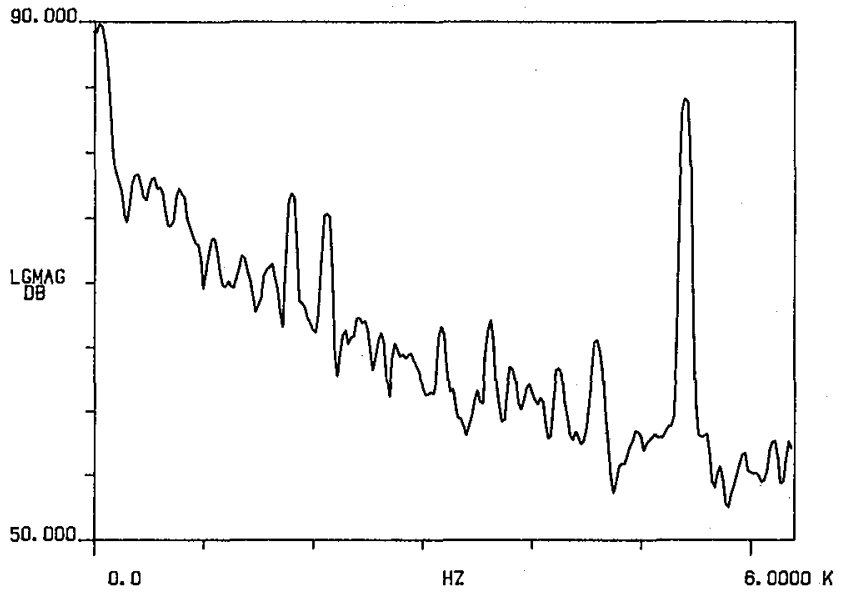

(b) Four bladed tail rotor

Fig. 8 Hughes 500D data at 80 knots airspeed..

Testing at the specified FAA flight conditions showed a cruise noise reduction of $3.2 \mathrm{db}$, an approach noise reduction of $0.6 \mathrm{db}$, and a takeoff noise reduction of $2.9 \mathrm{db}$.

Compressibility and blade vortex interaction effects from three different rotor blades were investigated through a series of tests performed in 1978 and 1979 by AVRADCOM with NASA assistance (reference 4). The three blades tested were the standard KAMAN $\mathrm{K} 747$, the KAMAN K747 wth an Ogee tip, and the Bell 540 (figure 9). These rotor systems were flown on an $\mathrm{AH}-1 \mathrm{~S}$ helicopter. The three rotors range from a square tip to a compound curved swept tip to give a good comparison of tip effects on noise generation. Sample impulsive noise data for the $60 \mathrm{knot}, 400 \mathrm{ft} / \mathrm{min}$ descent case is presented in figure 10 .

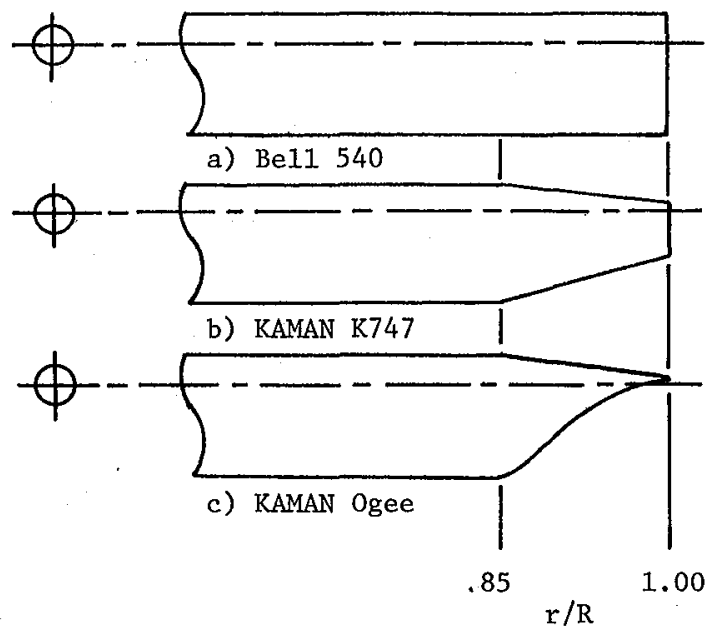

Fig. 9 AH-1S blade tip planforms.

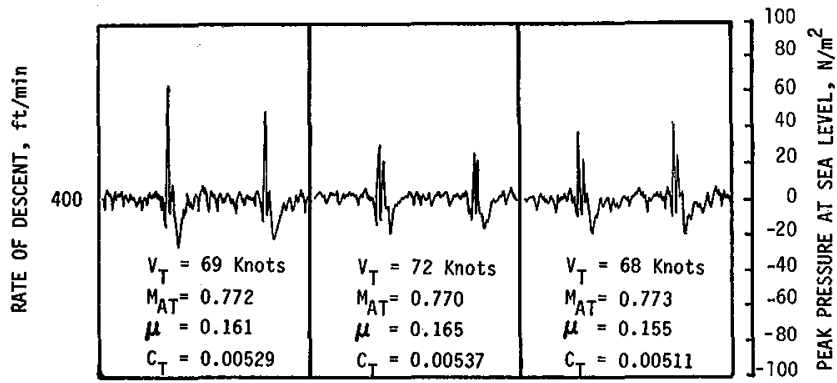
(a) 540
(b) $\mathrm{k} 747$
(c) OGEE

Fig. 10 All-1s time history data.

Notice that the change from a square tip to a swept tip results in a large change in the waveform, but a change in the two types of swept tips does not result in a large waveform change. This appears to indicate that the blade vortex interaction for the $\mathrm{K} 747$ and Ogee blades are not as strong as for the 540 blade.

In 1982 , as part of the acceptance flight test program of the Hughes AH-64 Apache, AVRADCOM requested NASA participation in obtaining flyover noise at the Army proving grounds, Yuma, Arizona. This test was conducted to determine if the Apache met the acoustic specificatons in the developement contract. As a target of opportunity, NASA also conducted air-to-air tests with the yo-3A. Regretably, this data is classified and is unavailable for publication at this time.

Perhaps the most extensive flight test program performed with the Y0-3A was the Tip Aerodynamic and Acoustics Test (TAAT). This test was flown during the summer of 1981 at NASA Ames Research Center on the extensively instrumented NASA AH-1G white Cobra (figure 11). 


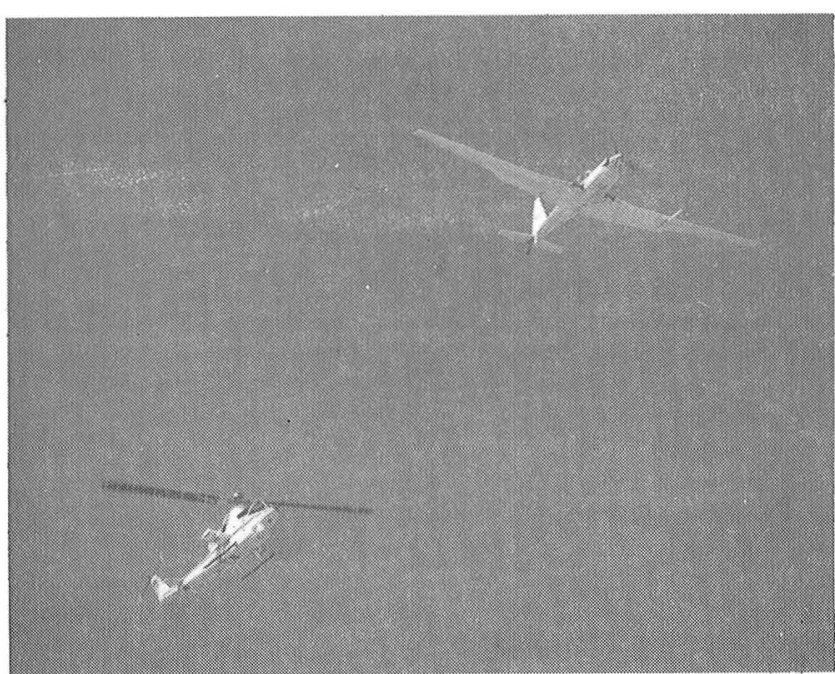

Fig. 11 NASA AH-1G and YO-3A in flight formation.

What made this a unique test was the simultaneous acquisition of extensive blade pressure data and acoustic data in an air-to-air testing configuration. One hundred and eighty eight pressure transducers arranged in eight radial stations, weighted in the tip region, gave a. fine grid of data on the rotor disk. The acoustic and pressure data are linked by a common time code and the transmitted one per rev signal recorded on the Yo-3A. Reduction of this data through the use of the Data Analysis and Management Package (DATAMAP) (reference 5) is being performed presently and will aid in the understanding of the acoustic phenomenon particular to helicopter flight.

\section{System Upgrades}

For any test facility used in engineering research to remain useful and productive, its capabilities must be continually improved. The YO-3A Acoustic Research aircraft is currently undergoing a multi-phase enhancement program. Included in the enhancements are a positioning system, increased data channe1s, and recording of the he1icopter parameters.

The visua1 positioning method currently used is adequate for certain tests but not highly satisfactory for precision research flight investigations. After a test, the general location of the aircraft is known but nothing quantitative has been recorded. Valuable as they are, it is not very satisfying having to rely on qualitative pilot comments. The helicopter Airborne Laser positioning System (HALPS), designed and built by Princeton University, is being developed to provide not on1y the quantitative recorded data of aircraft position, but to be more accurate than the current system. This added accuracy is obtained through a glideslope display used by the helicopter pilot which indicates deviation of the helicopter from the desired formation position. Continuous relative position data (radial separation, azimuth, and elevation) is recorded on the Yo-3A along with the acoustic data and aircraft parameters. This will allow the engineer to know to within +/- one foot the helicopter position relative to the YO-3A at all times. Using this ability, a flight test is being planned which will involve the helicopter flying within a spherical cone around the trailing flight test position. The goal of this test, with the help of HALPS, is to gain a more detailed understanding of helicopter acoustic directivity than was obtained during the initial $\mathrm{UH}-1 \mathrm{H}$ test conducted by the Army.

The fourteen channels currently available for recording are insufficient. Many of the data streams currently being recorded are not especial$1 \mathrm{y}$ demanding in terms of frequency response. Static pressure, for example, has not been known to go through high frequency gyrations. It seems rather wasteful to use one channel for each of these type data streams when several streams could be combined on one channel, thus freeing up channe1s for the more demanding types of data. To this end, methods such as FM multiplexing are currently being investigated.

During a test point, the acoustic signature of a helicopter varies significantly with control inputs. As the helicopter pilot pulls collective or makes sharp cyclic changes to maintain position, the rotor noise increases noticably. Time histories of control movements are thus a very important tool in the selection of which portion of data to analyze. Work is beginning on a system which will record these control positions of any test helicopter on the YO-3A tape recorder and be compatable with and use the existing one per rev link.

\section{$\underline{\text { Summary }}$}

The Y0-3A aircraft is used by NASA and Army personnel at Ames Research Center to conduct inflight acoustic testing of helicopters. This testing technique, proposed and pioneered by the Aeromechanics Laboratory, has proven itself to be an extremely valuable research tool in the understanding of helicopter related acoustic phenomenon. The in-flight testing technique eliminates most a11 the contaminating factors encountered in other types of external acoustic testing. To date, six flight tests have been conducted using this method, with eight specific tests scheduled for the future. In addition to these scheduled tests, the aircraft is available for testing "targets of opportunity" as they become available. This report has discussed the techniques used, tests conducted, current instrumentation, and instrumentation enhancements planned of the YO-3A aircraft. Future testing will lead to a more detailed understanding of helicopter acoustics and aid in the design of quieter rotorcraft.

\section{Acknowledgments}

The authors would like to acknowledge the cooperation and dedication of the research pilots who have flown in the YO-3A flight test programs over the years. Their willingness and support have ensured the taking of reliable data in a system whose accuracy depends to a large part on pilot ski11. The authors would also like to acknowledge F. H. Schmitz and D. A. Boxwe11 for their innovative ideas regarding the in-flight measurement of noise and for their drive in getting these ideas into a workable flight test tool. 
1. Schmitz, F. H. and Boxwe11, D. A., "In- flight Far Field Measurement of Helicopter Impulsive Noise," Journal of the American

Helicopter Society, Vol. 21 , No. 4, Oct. 1976.

2. George, R. E. and Duffy, V., "In-Flight Measurement of Aircraft Acoustic Signals," Proceedings of the 23rd Internationa1 Instrumentation Symposium, Vo1. 14, Las Vegas, Nev., 1977.

3. Splettstoesser, W. R., Schultz, K. J., Schmitz, F. H. and Boxwe11, D. A., "Model Rotor High-Speed Impulsive Noise," presented at the 39th Annual Forum of the American Helicopter Society, St. Louis, Mo., May 9-11, 1983.

4. Boxwe11, D. A. and Schmitz, F. H., "Full Scale Measurement of Blade-Vortex Interaction No1se," presented at the 36th Annual Forum of the American Helicopter Society, May 1980 .

5. PhIlbrick, R. B. and Eubanks, A. L., "Operational Loads Survey - Data Management System," Vol. 1, USARTL TR-78-52A, Jan. 1979. 


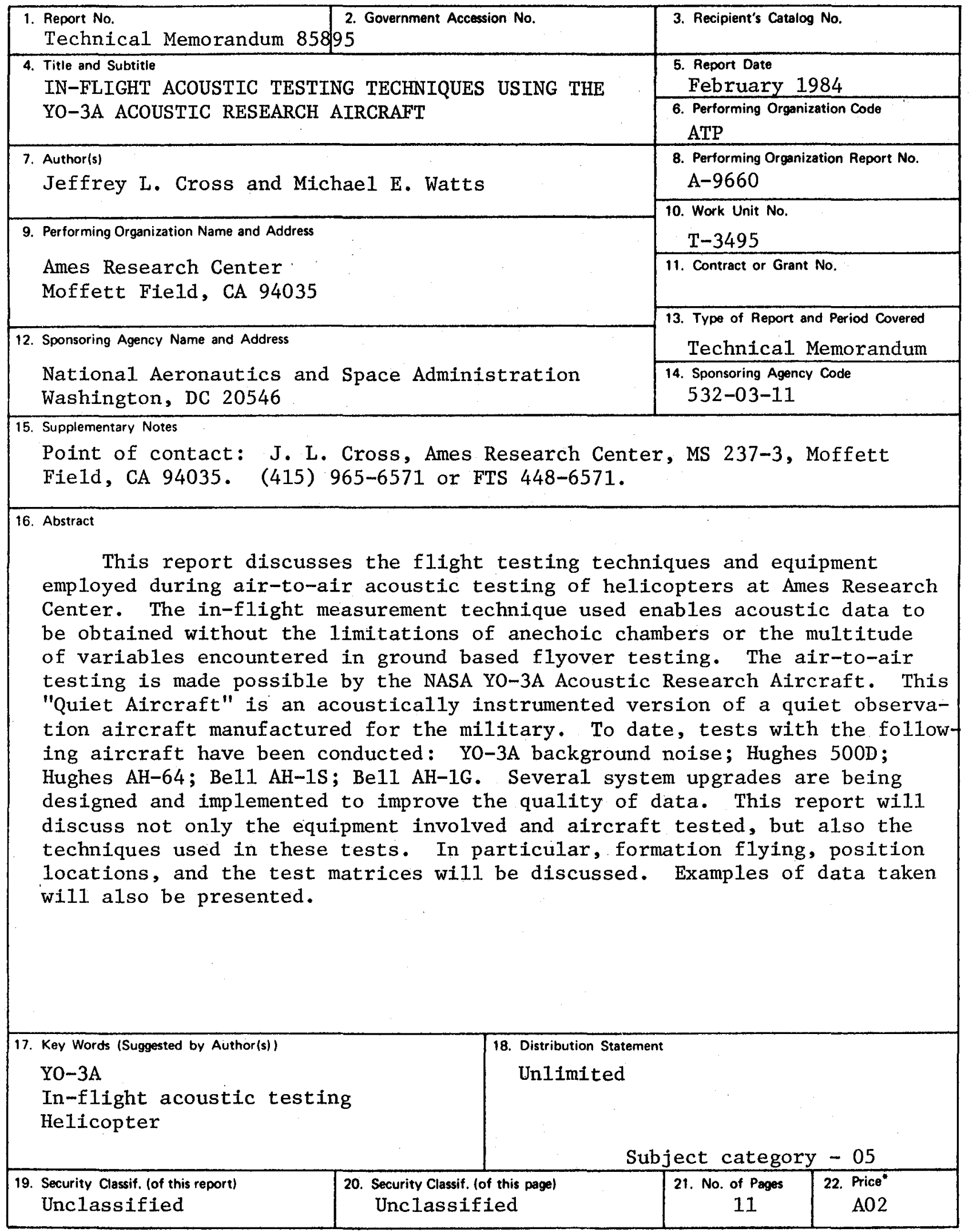

-For sale by the National Technical Information Service, Springfield, Virginia 22161 
